\title{
Desarrollo del programa familias con bienestar bajo el enfoque sistémico, construccionista*
}

\section{The "families with well-being" programme and the systemic constructionist approach}

\begin{abstract}
Nayibe Barreto Henao**
Carolina Yanguma Valentín

Universidad Santo Tomás, Colombia

Fundación Universitaria Konrad Lorenz

Recibido: 5 de mayo de 2014

Revisado: 22 de julio de 2014

Aceptado: 1 de agosto de 2014
\end{abstract}

\section{Resumen}

El presente artículo emerge de la experiencia como coordinadoras en el desarrollo del programa Familias con Bienestar, del Instituto Colombiano de Bienestar Familiar, en donde se emplea la visita domiciliaria, como dispositivo para la construcción del cambio y herramienta de intervención orientada a la práctica incluyente, discursiva, reflexiva y movilizadora de transformaciones en el sistema familiar, como una posibilidad para el desarrollo social, la promoción de comportamientos solidarios, de empoderamiento y de autogestión como requerimiento para la salud mental y emocional, comprendida desde el enfoque sistémico construccionista, en el escenario de la atención psicosocial con propósitos de la prevención de las violencias intrafamiliar, sexual y comunitaria.

Palabras clave: prevención, visita domiciliaria, enfoque sistémico-construccionista.

\section{Abstract}

This article results from the reflection on experience as coordinators in developing the "Familias con Bienestar" (Families with Well-being) program,

Artículo de investigación. Maestría en Psicología Jurídica, USTA, Colombia.

Correspondencia: Nayibe Barreto. Universidad Santo Tomás, Colombia. Fundación Universitaria Konrad Lorenz. Correo electrónico: nayibebarreto@hotmail.com. Dirección postal: Cra. 9 \# 51-11, Fac. Psicología, Bogotá, Colombia. 
sponsored by the Colombian Institute of Family Well-being, where the home visit is used as a device for the construction of change and as an intervention tool oriented to an inclusive discursive, reflective and mobilizing practice that leads to transformations in the family system, in itself as a possibility for social development, for promoting behavioral solidarity, empowerment and selfmanagement as a requirement for mental and emotional health, including a systemic constructionist approach on the stage of psychosocial care for purposes of prevention of domestic violence, sexual and community.

Keywords: Prevention, home visit, systemic constructionist approach.

\section{Introducción}

familias con Bienestar es uno de los programas en materia de prevención del componente familia y comunidad, del Instituto Colombiano de Bienestar Familiar (ICBF), el cual tiene como objetivo la prevención del fenómeno de las violencias intrafamiliar, infantil y sexual, en familias que se encuentran en condiciones de vulnerabilidad -definidas en el plan de gobierno del presidente Juan Manuel Santos como las personas y las familias que se encuentran en extrema pobreza-.

Con la intención de dar vía a la política de gobierno y a la misión institucional, el ICBF realiza la contratación de operadores con experiencia en el desarrollo de proyectos con familias y comunidades, razón por la cual la Asociación Profesionales de Colombia(APC) fue seleccionada para este encargo social y es la organización patrocinadora de esta investigación.

La relación construida entre el operador y el ICBF es el escenario donde se concilian los lineamientos, objetivos y alcance del programa para dar cumplimiento a políticas institucionales, locales y gubernamentales. Desde allí se establece la caracterización de la población beneficiaria, se definen los parámetros demográficos y socioeconómicos de los participantes, y se establece como principal requisito el deseo de participación y el interés de resolver asuntos emocionales en concordancia con los objetivos del programa.
En ese marco se dispone la visita domiciliaria como la acción preventiva, ya que permite la verificación de los modos de convivencia familiar y el control para el seguimiento del cumplimiento del programa.

Sin embargo, desde la lógica generativa que ofrece el enfoque sistémico-construccionista, se significó la visita domiciliaria como una herramienta de intervención, orientada a la práctica discursiva, reflexiva y movilizadora de las familias, y tal significación es el foco de análisis del presente artículo.

\section{Objetivos}

\section{Objetivo general:}

Reflexionar sobre la visita domiciliaria como un dispositivo de intervención en la movilización y transformación de las familias pertenecientes al programa Familias con Bienestar.

\section{Específicos:}

- Rescatar la visita domiciliaria como un dispositivo para la construcción de contextos de cambio.

- Develar la potencia del rol del interventor en los escenarios familiares.

- Potenciar la postura sistémico-construccionista como una oportunidad para el desarrollo de procesos de autogestión en las familias. 


\section{Bajo la mirada sistemico- construccionista}

intervenir las situaciones, historias y dilemas que afectan la calidad de vida de las personas, bajo un presupuesto de prevención, es un ejercicio que invitó a los profesionales del proceso a centrar su quehacer en una perspectiva paradigmática sobre la cual comprendieron, organizaron e interactuaron con las familias. Por lo tanto, este segmento está dedicado a evidenciar los principios del paradigma sistémico-construccionista complejo, que orientaron la labor de los profesionales vinculados al proyecto.

Desde la mirada sistémico-construccionista, se le concede gran importancia a la interacción en los fenómenos humanos, toda vez que conciben que "existe un tejido interdependiente, interactivo e inter-retroactivo entre el objeto de conocimiento y su contexto, las partes y el todo, el todo y las partes, las partes entre ellas" (Morin, 2001). Por lo tanto, los problemas no son generados por personas, sino en la interacción de estas, de los sistemas a los que pertenecen, y en ese sentido el proceso de intervención con las familias se convierte en un proceso interaccional, puesto que "ni la persona ni sus problemas existen en el vacío, sino que ambos están íntimamente ligados a sistemas recíprocos más amplios de los cuales el principal es la familia" (Maturana, 1980).

Operar desde esta postura implica reconocer que es en la interacción donde se construyen cambios, y es a partir del lenguaje donde los cambios emergen (Estupiñan, 2005). Por lo tanto, la interacción se genera desde la construcción de escenarios conversacionales, que dotan de sentido las situaciones donde se generan nuevos órdenes o significados.

Hablar de escenarios conversacionales, lenguajes posibilitadores de cambio e historias que dotan de sentido la realidad implica introducir las posturas construccionistas que reiteran que es en la interacción donde se construyen los cambios; por lo tanto, las intervenciones "no están compuestas de la acción individual, sino que son acciones conjuntas" (Shotter, 1993).
Dichas acciones conjuntas cobran sentido cuando se generan coordinaciones de significados entre los participantes, en un contexto intrínsecamente relacional; por lo tanto, la realidad se construye constantemente en la interacción, y es desde esta donde se generan acercamientos a las formas en que las personas crean sus realidades, significados y conocimientos. Es así como el consenso se convierte en una convención lingüística que determina lo que cuenta en la vida y cómo se cuenta.

Las historias que nos contamos son acciones sociales, que reiteran el protagonismo de las personas en sus realidades, en tanto que es desde la interacción donde construyen y deconstruyen situaciones e historias. Como lo resalta Ugazio (2001), "no existe una patología que no exprese las premisas culturales del contexto social, además del familiar en el que se desarrolla" (p. 40), ya que desde la complejidad se reconoce la influencia de otras esferas en la organización humana, como lo son la cultura, el Estado, la política, el contexto histórico y demás.

Desde la complejidad, se introduce la multidimensionalidad orientada a vislumbrar los fenómenos humanos como parte de un todo, interdependiente, interconectado, que evidencia cómo las dificultades y soluciones pueden ser vistas y transformadas en la relación: "todo desarrollo verdaderamente humano significa desarrollo conjunto de las autonomías individuales, de las participaciones comunitarias y del sentido de pertenencia con la especie humana" (Morin 2001). En ese sentido, Bronfenbrenner (1987) concibe que

la capacidad de un entorno para funcionar de manera eficaz como contexto para el desarrollo depende de la existencia de la naturaleza de las interconexiones sociales entre los entornos, lo que incluye la participación conjunta, la comunicación y la existencia de cada información en cada entorno con respecto al otro (p. 20).

La perspectiva sistémico-construccionista sobre la que se basó la intervención con las familias se convirtió en un instrumento para la transformación de los contextos, en cuanto se empleó como premisa central la concepción del cambio, concebida como un proceso interactivo donde las personas pasan 
de ser asistidas a ser partícipes en la búsqueda de nuevas realidades, que se generan en la trama narrativa y los interventores partícipes del tejido social construido. Más que expertos, se convierten en colaboradores que, desde el uso de narrativas, facilitan la activación de cambios y reconocen la particularidad de los procesos de las familias, así como la influencia directa que tienen los contextos en la co-creación de nuevas historias.

Desde la perspectiva de la horizontalidad de la relación, la validación de los escenarios de la cotidianidad en la que se movilizan las familias, su casa y su realidad se convierten en la fuente para la comprensión de sus historias y situaciones; todo esto con el ánimo de generar transformaciones complejas en los participantes, orientadas a

desplazarnos desde visiones ligadas a leyes generales y modelos normativos que configuran un mundo ordenado y predecible, hacia otras visiones donde las turbulencias, las oscilaciones y la novedad forman parte de lo cotidiano y de la construcción del cambio hacia un futuro abierto. (Fried Schnitman, 2004).

\section{Metodología: reposicionando la visita domiciliaria}

la visita domiciliaria surge en Europa, a finales del siglo XVIII e inicios del siglo xIX, como una actividad que permite asegurar que los 'pobres' reciban ayuda y como una oportunidad para el trabajo con 'desvalidos'. En Colombia, esta actividad empieza a implementarse a mediados del siglo xIx, cuando se estipula que la pobreza es un problema social de los cascos urbanos, y se plantea como estrategia la ayuda económica para estas personas (Castro, 2008)'

En ese momento histórico, la mirada institucional obedecía a criterios religiosos; las personas que podían acceder a esta ayuda económica debían disciplinarse en la doctrina católica, la cual exigía el cumplimiento del bautismo, el matrimonio y demás sacramentos para ser merecedores de la

1 Este texto es uno de los resultados del proyecto de investigación La filantropía en Colombia, 1870-1930, realizado en el marco del Grupo de Investigación Sociedad, Historia y Cultura, y que contó con el apoyo de Colciencias y la Universidad del Valle. ayuda, ya que eran vistas como virtuosas. Sin embargo, la demanda de ayuda se incrementó con el tiempo, por lo cual se incluyeron nuevos requisitos para recibir beneficios, tales como el número de hijos y las condiciones de miseria comprendidas desde el estado de la vivienda, características que podían explorarse y verificarse en la visita domiciliaria (Castro 2008).

Desde este recorrido histórico puede comprenderse la misión asistencial de la visita domiciliaria y cómo se fueron construyendo los lineamientos de tipo censal y de verificación de condiciones para la inclusión a lo que hoy puede denominarse programas. Bajo esta comprensión se rigidizan los procesos de atención psicosocial, a la vez que se generan roles claros frente a quién realiza la visita y quién es el visitado, tejiendo expectativas y rituales definidos bajo la lógica del cumplimiento de requisitos.

Hacia 1942 se empieza la formación técnica y profesional para esta labor; participaron la Universidad de Antioquia, del Rosario y el Colegio Mayor de Cundinamarca, además de la Javeriana en Bogotá y Cali, en un ejercicio que propendió por estructurar el servicio social, que más adelante se llamó trabajo social. De esta manera se fueron formando especialmente mujeres en la visita domiciliaria, la cual, culturalmente, aún se considera una acción desde la disciplina del trabajo social.

Desde esta disciplina se han hecho valiosos aportes a la psicología, con exponentes como Olga Silverstein, Virginia Satir, Ana María Campanini y Peggy Papp, Marianne Walters, Joseph Pérez, Amaya Ituarte Telleache, Ralph E. Anderson e Irl Carter. Si bien este estudio no discrimina las acciones de una u otra disciplina, resalta el encargo sociocultural que se delega desde la praxis o el imaginario institucional. Curiosamente, la literatura en torno a la visita domiciliaria desde la psicología es mínima, mientras que desde la disciplina del trabajo social abunda. En este sentido, es interesante que desde la disciplina de la psicología se suscite esta reflexión como una oportunidad para la integralidad, lo holístico y, por supuesto, lo transdisciplinario.

Cabe resaltar que en Latinoamérica se ha recurrido a la visita domiciliaria como estrategia para la superación de la pobreza, con unos resultados interesantes en términos del mejoramiento de la 
salud y la calidad de vida, sobretodo en países como Chile y Brasil, donde se ha propendido por el empoderamiento de las familias a través de la intervención en red, cuyo objetivo es la movilización de los recursos de la comunidad a favor de las familias (Donoso y Saldias, 1998).

A pesar de las gestiones que dan cuenta de la evolución de los objetivos de la visita domiciliaria, su comprensión se limita a una técnica que recoge elementos de la entrevista y la observación para el levantamiento de información, la cual posibilita la asistencia material, la ayuda técnica, la intención educativa, o como objetivo de evaluación y seguimiento, según lo describen Carzola y Fernández (2008), quienes diseñaron un modelo para la implementación de la técnica de la visita domiciliaria en Chile.

En Colombia se le da uso a la visita domiciliaria desde diferentes marcos institucionales, pero todos orientados a la asistencia o ayuda humanitaria, lo cual la convierte en un requisito para la participación en programas asistenciales, entre los que se encuentran Acción Social, Red Unidos, Instituto Colombiano de Bienestar Familiar, Secretaría de Integración Social, Secretaría de Salud, Salud Pública y Hospitales.

Darle uso a la visita domiciliaria como un dispositivo para la transformación de las familias implicó en los actores la deconstrucción de este modo dominante de comprender la visita y su objeto; es decir, pasar de la lógica asistencialista y de evaluación a una lógica participativa y de co-responsabilidad en la movilización de recursos personales, familiares y comunitarios, bajo la mirada sistémico-construccionista.

\section{Resultados: efectos de la visita domiciliaria bajo la mirada sistémico-construccionista}

\section{Proceso interventivo a través de la visita domiciliaria}

La intervención desde la visita domiciliaria se hace posible en la medida en que se afianza una relación participativa, caracterizada por la generación de acoples de contenido y emocionalidad que, desde la trama narrativa construida, permitieron fortalecer la emergencia de contextos dotados de sentido.

Frente a ello, se le concedió gran importancia a los relatos, los significados y el lenguaje, en tanto que orientaron la comprensión del cómo ha sido la construcción de las experiencias que hacen que las familias se narren a sí mismas, su historia y su contexto. Frente a ello, Maturana y Varela (1987) postulan que "en cualquier ser vivo el conocimiento no puede estar disociado de la existencia, ya que estos conocen, reconocen y se transforman en el devenir de la existencia" (p. 16).

Por lo tanto, el proceso terapéutico a partir de la visita domiciliaria se centró en generar procesos conversacionales circulares y movilizadores, encaminados a ampliar la trama narrativa y a identificar historias esperanzadoras y alternas en los relatos rígidos en los participantes. Dicha concepción hace que el valor del proceso interventivo sea visto desde la configuración de contextos conversacionales con sentido de transformación hacia el empoderamiento y la autogestión.

Por lo tanto, el escenario físico es solo el pretexto del encuentro conversacional y aquel lugar que facilitó la ritualización de la relación, concepción que contribuyó significativamente a visualizar la visita domiciliaria como un posible escenario para el proceso interventivo.

\section{La relación en la visita domiciliaria como dispositivo de cambio}

La visita domiciliaria posibilitó la construcción de una relación horizontal, donde la familia y el interventor construyeron cercanía y cooperación en doble vía, caracterizada por el mutuo cuidado, ya que las familias, como conocedoras del territorio, generaban acciones de prevención hacia los interventores frente a los posibles riesgos, ya conocidos por ellos, de la misma manera que los terapeutas disponían de un discurso desde la prevención de riesgos para las familias. Por lo tanto, 
se fusionaban dos versiones de prevención en una sola intención de cuidado y de solidaridad, que terminó por gestar movilizaciones de empoderamiento y de miradas recursivas y participativas.

La visita domiciliaria facilitó la pragmática de la práctica discursiva, en tanto que las familias lograron descongelar el discurso de la vulnerabilidad y la necesidad de cuidado, al posicionarse como cuidadores de los interventores, quienes reconocían su fragilidad en contextos ajenos a su cotidianidad. Pero al mismo tiempo, los interventores, bajo la mirada del modelo solidario, eran potentes en el cuidado de la permanencia de la familia y en la exaltación de sus recursos.

Se lograron procesos co-laboradores, co-constructivos $\mathrm{y}$, por ende, transformadores, movilizadores de esperanza, pues cada acción redundó en el mensaje de cambio frente a su propia fortaleza y protagonismo. Por lo tanto, la visita domiciliaria es terapéutica en sí misma, pero bajo la mirada sistémico-construccionista con sentido de transformación, ya que es una acción consensuada, de reconocimiento y potente en la manifestación de los recursos, donde la experiencia vivida permite que las familias movilicen sus relatos y significados, y con ello potencien el cambio.

\section{Lo recursivo de la visita domiciliaria}

La recursividad se convierte en la conexión "permanente de los niveles y dominios de la información" (Estupiñan, 2006), que empleados en el contexto de la visita domiciliaria, permitieron en los actores la comprensión y movilización de los significados que dotan de sentido la configuración de los dilemas en las familias. Desde allí, el interventor se ve en la necesidad de generar diversas prácticas y estrategias que le posibiliten construir dichos grados de conexión.

En ese sentido, la visita domiciliaria se concibió como estrategia en sí misma, en tanto que el simple acto de realizar un acompañamiento en la cotidianidad familiar es significado como una acción de inclusión en personas que tienen como narrativa dominante la exclusión social; este hecho genera en los participantes una sensación de pertenencia con la ciudad, y afianza en ellos la visión de ser importantes en la construcción social.

Por otro lado, el interventor recurre a su creatividad, la cual emerge en el aprovechamiento de sus recursos y los que le ofrece el contexto, para posicionarse como co-narrador en la cotidianidad de los escenarios en que se movilizan las familias, ya que direcciona su acompañamiento en el reconocimiento de dispositivos de cambio, enfocándose en los recursos familiares y sociales, más que en el déficit, que hace parte de los relatos cristalizados de las familias.

El acto de co-narración es posible cuando en la relación construida entre las familias y los interventores se generan vínculos cohesionados que permiten introducir tramas narrativas alternas que connotan la realidad, el destino y la lógica de la vida, de un modo generativo y reinventante de la vida misma.

Otro aspecto por resaltar fue la apertura del interventor en la vinculación a la cotidianidad de las familias, lo cual le permitió generar una comprensión contextual, ya que en algunos momentos la descripción de las familias dista del imaginario del interventor; así la descripción sea detallada y rica en la transmisión de sensaciones, este se queda corto frente a la vivencia o inmersión en el contexto mismo.

La visita domiciliaria se convierte en un acto recursivo desde la postura eco-sistémica, toda vez que se orienta a la valoración de los procesos humanos en contextos connotados como de sufrimiento, que para ser movilizados requieren de la construcción de un vínculo sólido en los actores. Asimismo, implica la creatividad del interventor y el reconocimiento de sus herramientas personales para la consolidación de cambios propositivos y la activación de la capacidad de resiliencia en las familias, las comunidades y los interventores. 


\section{Los retos}

Para el empleo de la visita domiciliaria como dispositivo de cambio, se concibe como gran reto la deconstrucción del modo dominante de comprenderla, es decir, movilizar la versión de una estrategia para la recolección de información hacia una herramienta que posibilita la construcción de sentidos alternos en la búsqueda del cambio personal, familiar y social.

Otro reto que atañe al interventor es la manera en que se posiciona, ya que deja de lado el estilo directivo y su posición de poder, para avanzar en la construcción de relaciones horizontales, donde se reconoce el papel protagónico de las familias en la movilización del cambio. Por lo tanto, es necesario que el interventor haga meta-observaciones sobre el vínculo generado y calibre de manera permanente las expectativas de la relación construida, pues la visita domiciliaria genera implicaciones en la dinámica familiar; es una intrusión consentida en la cotidianidad del otro.

Desde una óptica más amplia, la visita domiciliaria como estrategia de intervención invita a transformar la atención psicosocial en procesos de desarrollo comunitario, para atender a las necesidades particulares de las familias. Desde allí se sugiere un desafío para el profesional de las ciencias humanas, en la medida que estimula a crear y re-crear estrategias de intervención que convoquen lo mejor del ser humano, así como sugerir una movilización de posturas institucionales y políticas. Institucionales, porque implica moverse del agente acusador al agente de ayuda, frente a la consolidación de procesos humanos, solidarios y propositivos; es poner a la institución en función social, en función de las familias y de la comunidad, volcar el estatus; la prioridad es la humanidad y no la institución.

Se trata no de que las familias estén en función de instituciones que las vigilen y controlen, con miedo y cumpliendo requisitos para ser incluidas en programas de ayuda, sino de que la institución esté a favor del empoderamiento, de la autogestión y la sostenibilidad de aquellas. Esta diferencia hace que se construya una sociedad tolerante, autogestora y, por supuesto, responsable de sus decisiones, capaz de abrir sus casas y sus contextos para la transformación positiva de su realidad.

\section{Conclusiones}

Desde la perspectiva eco-sistémica, la visita domiciliaria se convierte en un dispositivo para la construcción de contextos de cambio, en tanto que es una acción consensuada entre los participantes (familias e interventores), orientada a la inclusión y participación en la transformación de contextos cuyos relatos dominantes incluyen el déficit y la desesperanza, puesto que la percepción en el sujeto, la familia y la comunidad es de desempoderamiento de sus recursos, oportunidades y posibilidades.

Por lo tanto, la movilización de las familias y de los contextos en los que se tejen las narrativas de conflicto o de violencia incrementa la relevancia de la psicología sistémica-construccionista como una posibilidad para el desarrollo social, para la promoción de comportamientos solidarios, de empoderamiento y de autogestión como requerimiento para la salud mental y emocional.

Se resalta la potencia de la visita domiciliaria como escenario cotidiano que posibilita lo nuevo, la construcción de nuevos relatos que tienen lugar en la relación participativa, de cooperación y horizontalidad que se teje entre los actores y que es movilizadora en sí misma, puesto que introduce cambios en los contenidos narrativos y pragmáticos de la relación. Por ejemplo, emerge la inclusión en un contexto excluido y se fortalecen las capacidades de cuidado en una población que se lee como "desvalida", pero que termina protegiendo los interventores que, bajo la óptica del cuidado, reconocen su vulnerabilidad en contextos desconocidos y con dinámicas diferentes a las del consultorio privado. 
El aporte de los programas de prevención desde esta lógica es una ganancia en varios sentidos: primero, la garantía de la salud emocional como principal recurso de la salud física, lo cual sugiere una menor demanda de recursos hacia la rehabilitación y la sanación; segundo, la garantía en el aseguramiento de poblaciones autogestoras y empoderadas de su realidad requiere menos recursos para programas de subsidiariedad de ayuda en la sobrevivencia; tercero, una sociedad que invierte en la recuperación del tejido social es una sociedad con menor riesgo de violencia intrafamiliar, infantil, sexual o comunitaria.

Por último se resalta el reconocimiento de las ciencias humanas desde la comprensión sistémicaconstruccionista como una herramienta útil, concreta y asertiva para la configuración de procesos sociales, reflexivos y canalizadores de cambio.

\section{Referencias}

Bronfenbrener, U. (1987). Ecología del desarrollo humano. Barcelona: Paidos

Carzola, K., y Fernández, J. (2008). Reflexiones en torno a la visita domiciliaria como técnica de trabajo social. Santiago de Chile.

Castro, B. (2008, junio). Las visitas domiciliarias femeninas en Colombia. Sociedad y Economía, (14), 109-131.
Donoso, M., y Saldias, P. (1998). Modelo de intervención para el trabajo social familiar. Santiago de Chile: Universidad Católica de Chile.

Estupiñan, J. (2005, julio-diciembre). Psicoterapia sistémica, psicología y responsabilidad social: La hipótesis de la convergencia entre sabiduría y conocimiento técnico. Diversitas, 1(2), 227- 237.

Estupiñan, J. (2006). Historias y narrativas familiares en diversidad de contextos. Dossier (2). $22-28$

Fried Schnitman, D. (2004). Perspectivas e instrumentos generativos en psicoterapia. Buenos Aires: Sistemas Familiares.

Maturana, H. (1980). Desde la biología a la psicología. Santiago de Chile: Universitaria.

Maturana, H., y Varela, F. (1987). El árbol del conocimiento. Santiago de Chile: Debate.

Morin, E. (2001). Introducción al pensamiento complejo. Madrid: Gedisa.

Presidencia de la República. Plan nacional de desarrollo 2010-2014. Recuperado de www.dnp. gov.co

Shotter, J. (1993). Realidades conversacionales. Madrid: Amorrortu.

Ugazio, V. (2001). Historias permitidas, historias prohibidas. Barcelona: Paidós. 